\title{
Research about Ocular Region Automatic Outline Fit
}

\author{
Hong $\mathrm{BaO}^{1, \mathrm{a}}$ and Ming $\mathrm{Li}^{1, \mathrm{~b}}$ \\ ${ }^{1}$ School of Computer and Communication Engineering \\ University of Science and Technology Beijing Beijing 100083 China \\ abaohong@ies.ustb.edu.cn, bliming_pr@163.com
}

Keywords: Bezier curve, control point, convex hull, feature extraction

\begin{abstract}
In recent years, with the development of artificial intelligence, more experts and scholars have joined in the research about facial expression recognition. Algorithms of feature extraction and facial expression recognition are put forward in succession, and each has advantages and disadvantages. A research about feature extraction method and facial expression recognition recently put forward which is on the basis of Bezier curve found that representation of expression by geometrical characteristics of Bezier curve control points has the advantages of small characteristic dimension and rapid arithmetic speed, but the point selection needs to be marked manually, which can't meet the requirements of real time recognition of facial expression and can't ensure the consistency of several-time marks. Ocular region automatic outline fitting and features extraction method was put forward under such conditions, extracting convex hull area of eye outline by image processing techniques and Bezier curve fitting and control points obtaining by interpolation methods. Japanese female facial expression database (JAFFE). All images after several tests could gain favorable fitting effect and the data extracted was kept in consistency.
\end{abstract}

\section{Introduction}

In recent years, with the high development of artificial intelligence, experts and scholars in artificial intelligence fields have paid more and more attention to the issue of facial expression recognition of pictures or videos. Various researches are aiming at improving facial expression recognition effectiveness, automatic identification and accuracy.

During facial expression recognition process, effective extraction of facial features is an important step for facial expression recognition. Facial expression information mainly shows in changes of eyebrows, eyes, nose and mouse and mutual relations between these changes. A research about feature extraction method and facial expression recognition [1] have been put forward recently. The article presented that marking the main facial organs by Bezier curve, obtaining the curve control points through inverse determination technology of Bezier curve control points [2], and take advantage of the relationship between each other, such as slope, distance, etc., could achieve large reduction of arithmetic quantity as well as recognition rate guarantee. It is verified by testing that this method could obtain a favorable recognition rate.

However, according to the theory, outline points need to be marked manually before calculating Bezier curve control points, so features cannot be extracted automatically. Under such a status, this paper put forward ocular region automatic outline fitting and features extraction method. It is verified that the obtained feature information meets the subsequent facial expression recognition requirements by curve marks comparison and error calculation of control point coordinate.

According to the features of cubic Bezier curve, can extract the facial expression shape feature integrally. One smooth curve could be accurately depicted by four control points and thereby the shape features of facial expression key parts, such as eyes, eyebrows and mouth, etc., are described and extracted by the whole curve. Its advantages are: Morphological characteristics of face organ are identical to features and the property of Bezier curve. Each organ can be fitted into one or more smooth curves by cubic Bezier curve. In general feature extraction of local part, sufficient disperse curve points are required to accurately depict organ curve and then make a classification according to the relevant positions of curve points. In this method, accurate point tracing of curve points is 
required, which makes the calculation complex and inaccurate. In contrast, cubic Bezier curve just needs four control points to accurately depict one organ curve for integral grasp of organ features. This method doesn't need accurate point tracing, thus facial expression classification error as a result of point tracing error is dramatically reduced. Control point changes of cubic Bezier curve depicting organs as a result of facial expression changes are sufficient to identify facial expression classification.

According to the definition of Bezier curve, cubic Bezier curve is defined as Eq.1.

$$
P_{0}^{3}=(1-t)^{3} P_{0}+3 t(1-t)^{2} Q_{1}+3 t^{2}(\mathbf{1}-\boldsymbol{t}) Q_{2}+t^{3} P_{3}
$$

In the Eq.1, $P_{0}^{3}$ is the point of the Bezier curve. The four points on Bezier curve are $\mathrm{P}_{0}, \mathrm{Q}_{1}, \mathrm{Q}_{2}$ and $\mathrm{P}_{3}$ which determine the control points. An identical equation is obtained by substituting the $\mathrm{P}_{0}$ and $\mathrm{P}_{3}$ into Eq.1. Therefore, substituting the rest two points in Eq.1 will get:

$$
\left\{\begin{array}{l}
P\left(t_{1}\right)=\left(1-t_{1}\right)^{3} P_{0}+3 t_{1}\left(1-t_{1}\right)^{2} Q_{1}+3 \boldsymbol{t}_{1}^{2}\left(1-t_{1}\right) Q_{2}+\boldsymbol{t}_{\mathbf{1}}^{3} P_{3} . \\
P\left(t_{2}\right)=\left(1-t_{2}\right)^{3} P_{0}+3 t_{2}\left(1-t_{2}\right)^{2} Q_{1}+3 \boldsymbol{t}_{2}^{2}\left(1-t_{2}\right) Q_{2}+\boldsymbol{t}_{2}^{3} P_{3} .
\end{array}\right.
$$

According to the approximate evaluating method of the value of $t$ in the paper:

$$
\left\{\begin{array}{l}
t_{1}=\left|P_{0} Q_{1}^{\prime}\right| /\left|P_{0} P_{3}\right| \\
t_{2}=\left|P_{0} Q_{2}^{\prime}\right| /\left|P_{0} P_{3}\right|
\end{array}\right.
$$

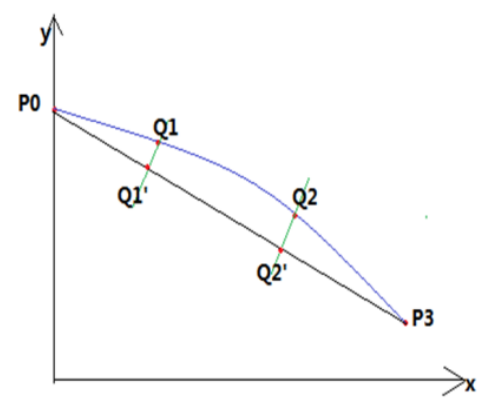

Fig.1 Four points on curve are $\mathrm{P}_{0}, \mathrm{Q}_{1}, \mathrm{Q}_{2}$, and $\mathrm{P}_{3}$

In Fig $1, \mathrm{Q}_{1}^{\prime}$ and $\mathrm{Q}_{2}^{\prime}$ are the pedals of $\mathrm{Q}_{1}$ and $\mathrm{Q}_{2}$. Point coordinates of control points $\mathrm{Q}_{1}$ and $\mathrm{Q}_{2}$ can be obtained by unifying Eq.2 and Eq.3.

Through the above steps, the control points can be obtained. From the above-mentioned contents, cubic Bezier curve needs four points on line to accurately simulate an organ outline curve of face features. Several tests have verified the feasibility of feature extraction on the basis of Bezier curve.

In conclusion, facial expression feature extraction on the basis of Bezier curve can effectively depict features of facial organ, to conduct the subsequent facial expression classification. But in extraction of points on curve, which are P0, P1, P2 and P3, artificial marks on the outline of eyebrows, eyes and mouth are required, including the endpoints of outline ends. Automatic extraction can't be achieved, so that the application of the method in facial expression recognition is restricted. And also, as a result of the influence of multi factors in artificial marks and point selection, data consistency for control points extracted in multi testing for the same image can't be ensured. Under such conditions, ocular region automatic outline fitting and features extraction method is put forward to improve the automaticity of feature extraction and ensure consistency of multi tests.

\section{Research work}

Eyes features are important in facial expression recognition, different expressions will cause different geometrical characteristic change of eyes. Aiming at yellow race, the contrast of complexion between eyes and face is intense. In grayscale images, the information of eyes can be well marked by the 
shadow outline of eyeballs and eyes, so the morphological characteristics of eyes can be extracted by applying the method of image processing. However, there will be great deviation between the directly exacted effect and expected effect since the influences of illumination, hair, vein and other factors. The image processing technology should be used to weaken the interference brought by noise, at the same time, optimize the information extracted by using geometry characteristics of eyes. The binary image used indicate the approximate outline of eye by using convex hull of polygon; the extraction of cubic Bezier curve fitting and control point to eye outline can be finished after calculating the interpolation. The general algorithm flow is shown as Fig.2.

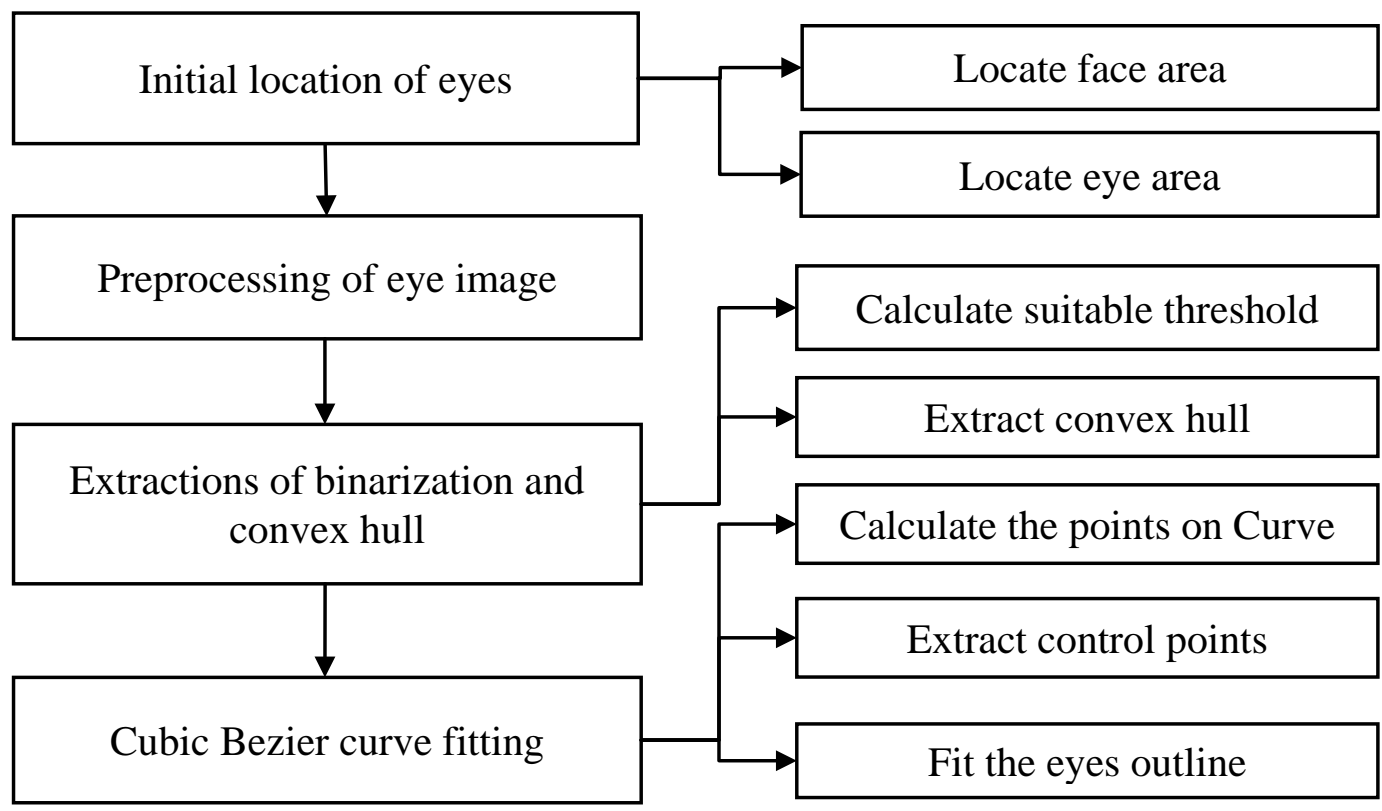

Fig. 2 Algorithm flow

Initial location of eyes. Based on prior knowledge, facial image generally includes background, facial organs, hair, neck and other factors, the parse tree based on classification and regression (CART) should be firstly applied to locate human face for excluding the influence of hair and background on the subsequent initial location of eye. [3, 4]

After obtaining the face area, the face area shall be divided into two portion, upper face and lower face. Since the localization effect of eyes will be influenced by mouth and nose, the eye detection can be conducted after the completion of partitioning. [5, 6] False detection will happen since the similar morphological characteristics of eyebrow and eye are influenced by illumination intensity, initial location of eye can be completed by selecting the location close to lower edge of upper face as eye area according to the distribution of the eyebrow and eye, as shown in Fig. 3. Partition the initial location area as left eye part and right eye part.

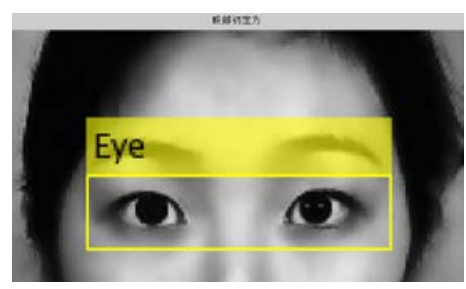

Fig. 3 Initial location of eye

Preprocessing of eye image. Image preprocessing is mainly aimed for eliminating noise and enhancing the gray contrast degree of region of interest. Now there are many such methods, among which, the adaptive smooth filtering can only smooth local area of noise and doesn't smooth the non-noise local area and then minimum the influence of blur. Gamma transformation can enhance the contrast of low gray area according to the parameters. [7] So they are selected as preprocessing methods according to the test effect, and the obtaining effect meets the requirement of experiment. 
Extractions of binarization and convex hull. The extractions of binaryzation and convex hull aiming at images after completing preprocessing can obtain the data information of region of interest of eyes, to do the subsequent operation of curve fitting and adapt the problem of inconsistency of multiple images' gray level. Here, propose the binaryzation of changing threshold.[8]

Binaryzation of changed threshold. Relative few images will be obtained after completing image preprocessing; the colors of eyes and complexion of yellow race have great differences according to the priori knowledge, so in the grayscale images, the gray value of eye is relative low and the gray values of outline and eyeball are between 1 and 80 while the gray value of complexion is high, which is between 80 and 255; there will be some errors between the images after binaryzation and excepted images since the shadow produced by illumination and gray value around outline line, so obtained binary image should be corrected according to the geometrical characteristic of eye area and the binaryzation of changing threshold was proposed:

The stipulated initial threshold is 0.3 ; perform binaryzation to grayscale images.

Extract connected component of binary images.

According to observation, eye area is mainly concentrated in the central area of left and right eye areas, set filter array, select connected component that has most hit points in central area and on this standard to eliminate the noise component around that is too small and obtain the optimized binary image.

Extract convex hull points by using the above-mentioned obtained images and calculate the width of region of interest according to the convex hull points; here the eye size is set as 25 , if the width of region of interest is less than 25 , it means that the threshold value is too small and the initial threshold should be added and then continue to perform the step in 1); Otherwise, the threshold value is suitable and the binarization segmentation is completed.

Binary image is as shown in Fig. 4.

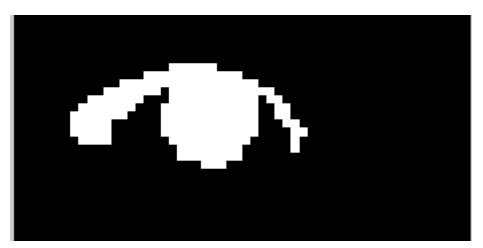

Fig. 4 Binarization image of left eye area

Convex hull extraction. Convex hull can be regarded as the boundary of minimum convex polygon of all vertices in closing points set $S$, its precise definition is as follows: suppose that set $S$ is the set consisted by $k$ points in $n$ dimensions space, namely $S=\{x 1, x 2, \ldots x k\}$, $x i$ is $n$ dimensions vector. Definite convex hull Conv(S) of S as:

$$
\operatorname{Conv}(\mathrm{S})=\left\{\mathrm{x}=\lambda_{1} x_{1}+\lambda_{2} x_{2}+\cdots+\lambda_{k} x_{k} \mid \lambda_{1}+\lambda_{2}+\cdots+\lambda_{k}=1\right.
$$

Convex hull points indicate the vertices of convex hull polygon; the region of interest of eyes can be obtained after completing images binaryzation and the outline of eyes can be represent approximately by extracting the convex hull of it, as shown in Fig.5.

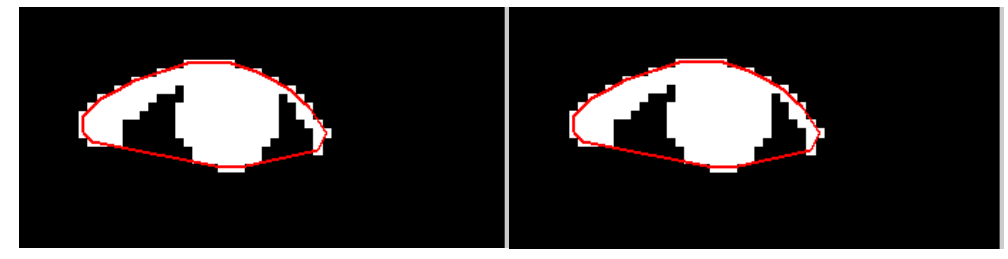

Fig. 5 Convex hull image of left eye area(left); Convex hull points image of left eye area(right)

Cubic Bezier curve fitting. Cubic Bezier curve fitting is to select the suitable points on line and calculate the fitting curve through cubic Bezier curve fitting formula and then acquire the control points by means of inverse principle of Bezier curve, the main steps include: location of points on line determination, linear interpolation, curve fitting and control points obtaining. 
Position selection and determination of points on line. Cubic Bezier curve can be fitted according to four points on line and obtain the four control points. However, it is showed by the tests that, for the same curve, except for left and right endpoints, lateral selection positions of two points on line in the middle of the curve have a great effect on the shape of the curve. It is showed that the fitting effect of the shape of curve reached the best when two points on line in the middle area are distributed on the curve evenly, thus, we should execute the lateral trisection of convex hull point obtained, and the two points on line in the middle area are separately on the $1 / 3$ and $2 / 3$ place.

Linear interpolation. According to the extracted convex hull point image, the convex hull may not exist when selecting position in the desire line, which needs to conduct linear interpolation. The method is: calculate lines formula of left and right convex hull point nearest to the expected selective points, calculate ordinate of interpolating point according to the abscissa about selection position of points on line; in other cases, under the condition of closing eyes slightly, if no convex hull points exist under the eyelid part, linear interpolation should be conducted according to the connecting lines of left and right endpoints. It is showed on the basis of tests that the interpolation conducted by using this method has a good effect on the curve fitting and meets the requirements on subsequent classification.

Curve fitting and control point calculation. Taking left and right endpoints' connecting line as the parting line, the convex hull point obtained from region of interest of eyes divides the region into upper and lower eyelid. The upper and lower eyelid should use data points obtained by convex hull point and interpolation as four points on line of fitting cubic Bezier curve, and fit and find control point making use of Bezier curve fitting formula and reserving control point method mentioned in the introduction.

\section{Experimental analysis}

This experiment chooses Japanese female facial expression database, which is called JAFFE database for short. There are 213 expression images in the facial expression database, including 6 different kinds of expressions of 10 Japanese females (including 7 kinds of neutral expressions), and every expression of each person includes 3-4 images for a total of 214 images. As shown in Fig. 6, it displays some different images in the JAFFE database.

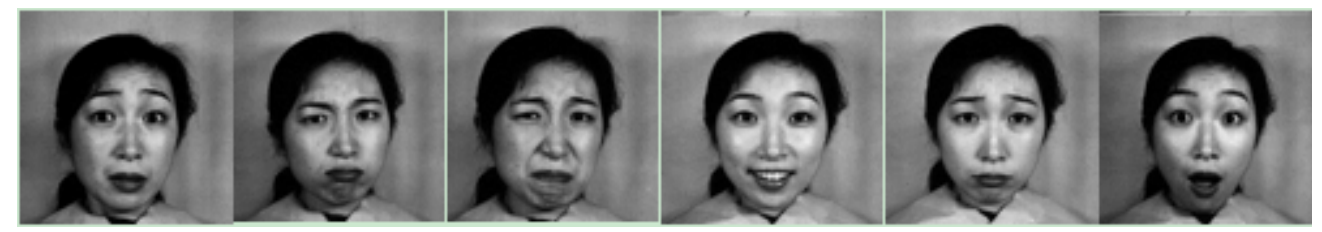

Fig. 6 Image show with different expressions in the JAFFE database

Image contrast between auto mark and manual mark on neutral expression is as shown in Fig. 7.

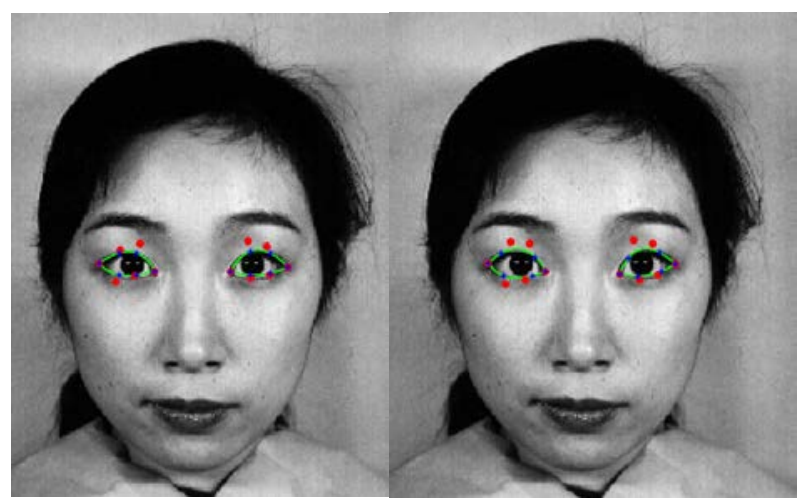

Fig. 7 Contrast diagram on auto mark (left) and manual mark (right) of neutral expression

represents control point; * represents points on line; curve represents eye outline.

Control point coordinate contrast on neutral expression of auto mark and manual mark is as shown in Table 1 and Table 2. 
Table 1 Coordinate contrast on Left Eye

\begin{tabular}{|c|c|c|c|c|c|c|c|}
\hline \multirow{2}{*}{ Left Eye } & \multicolumn{2}{|c|}{ Manually } & \multicolumn{2}{c|}{ Automatic } & \multicolumn{2}{c|}{ Error[\%] } \\
\cline { 3 - 8 } & $\mathrm{X}$ & $\mathrm{Y}$ & $\mathrm{X}$ & $\mathrm{Y}$ & $\mathrm{X}$ & $\mathrm{Y}$ \\
\hline \multirow{2}{*}{ Points of the Corner of Eye } & $\mathrm{P} 0$ & 83 & 128 & 83 & 127 & 0.00 & 0.78 \\
\cline { 2 - 8 } & $\mathrm{P} 3$ & 112 & 131 & 111 & 131 & 0.89 & 0.00 \\
\hline \multirow{2}{*}{ Upper Eyelid Control Points } & $\mathrm{P} 1$ & 94 & 117 & 93 & 120 & 1.06 & -2.56 \\
\cline { 2 - 8 } & $\mathrm{P} 2$ & 104 & 117 & 103 & 117 & 0.96 & 0.00 \\
\hline \multirow{2}{*}{ Upper Eyelid Points on Curve } & $\mathrm{Q} 1$ & 90 & 122 & 93 & 122 & -3.33 & 0.00 \\
\cline { 2 - 8 } & $\mathrm{Q} 2$ & 107 & 124 & 102 & 122 & 4.67 & 1.61 \\
\hline \multirow{2}{*}{ Lower Eyelid Control Points } & $\mathrm{P} 1$ & 92 & 138 & 91 & 136 & 1.09 & 1.45 \\
\cline { 2 - 8 } & $\mathrm{P} 2$ & 102 & 136 & 101 & 133 & 0.98 & 2.21 \\
\hline \multirow{2}{*}{ Lower Eyelid Points on Curve } & $\mathrm{Q} 1$ & 91 & 134 & 93 & 133 & -2.20 & 0.75 \\
\cline { 2 - 8 } & $\mathrm{Q} 2$ & 105 & 134 & 102 & 133 & 2.86 & 0.75 \\
\hline
\end{tabular}

Table 2 Coordinate contrast on Right Eye

\begin{tabular}{|c|c|c|c|c|c|c|c|}
\hline \multirow{2}{*}{ Right Eye } & \multicolumn{2}{c|}{ Manually } & \multicolumn{2}{c|}{ Automatic } & \multicolumn{2}{c|}{ Error[\%] } \\
\cline { 3 - 8 } & $\mathrm{X}$ & $\mathrm{Y}$ & $\mathrm{X}$ & $\mathrm{Y}$ & $\mathrm{X}$ & $\mathrm{Y}$ \\
\hline \multirow{2}{*}{ Points of the Corner of Eye } & $\mathrm{P} 0$ & 150 & 133 & 150 & 131 & 0.00 & 1.50 \\
\cline { 2 - 8 } & $\mathrm{P} 3$ & 178 & 128 & 179 & 129 & -0.56 & -0.78 \\
\hline \multirow{2}{*}{ Upper eyelid Control Points } & $\mathrm{P} 1$ & 156 & 116 & 158 & 116 & -1.28 & 0.00 \\
\cline { 2 - 8 } & $\mathrm{P} 2$ & 166 & 117 & 168 & 118 & -1.20 & -0.85 \\
\hline \multirow{2}{*}{ Upper Eyelid Points on Curve } & Q1 & 154 & 125 & 160 & 121 & -3.90 & 3.20 \\
\cline { 2 - 8 } & $\mathrm{Q} 2$ & 170 & 122 & 170 & 122 & 0.00 & 0.00 \\
\hline \multirow{2}{*}{ Lower Eyelid Control Points } & P1 & 160 & 136 & 159 & 135 & 0.63 & 0.74 \\
\cline { 2 - 8 } & P2 & 169 & 133 & 169 & 133 & 0.00 & 0.00 \\
\hline \multirow{2}{*}{ Lower Eyelid Points on Curve } & Q1 & 134 & 160 & 133 & 158 & 0.75 & 1.25 \\
\cline { 2 - 8 } & Q2 & 132 & 170 & 132 & 165 & 0.00 & 2.94 \\
\hline
\end{tabular}

Image contrast of auto mark and manual mark on surprise expression is as shown in Fig. 8.

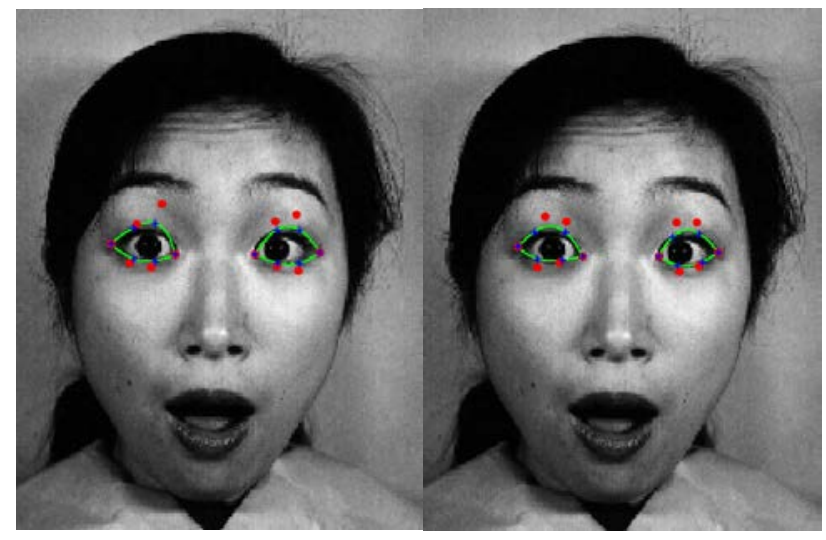

Fig. 8 Contrast diagram of auto mark (left) and manual mark (right) of surprise expression - represents control point; * represents points on line; the curve represents eye outline

Coordinate contrast on control point and points on line of auto mark and manual mark is as shown in the following Table 3 and Table 4.

Table 3 Coordinate contrast on Left Eye

\begin{tabular}{|c|c|c|c|c|c|c|c|}
\hline \multirow{2}{*}{ Left Eye } & \multicolumn{3}{c|}{ Manually } & \multicolumn{2}{c|}{ Automatic } & \multicolumn{2}{c|}{ Error[\%] } \\
\cline { 3 - 8 } & & $\mathrm{X}$ & $\mathrm{Y}$ & $\mathrm{X}$ & $\mathrm{Y}$ & $\mathrm{X}$ & $\mathrm{Y}$ \\
\hline \multirow{2}{*}{ Points of the Corner of Eye } & $\mathrm{P} 0$ & 83 & 127 & 82 & 127 & 1.20 & 0.00 \\
\cline { 2 - 9 } & $\mathrm{P} 3$ & 113 & 132 & 111 & 132 & 1.77 & 0.00 \\
\hline \multirow{2}{*}{ Upper Eyelid Control Points } & $\mathrm{P} 1$ & 96 & 113 & 93 & 118 & 3.13 & -4.42 \\
\cline { 2 - 9 } & $\mathrm{P} 2$ & 105 & 116 & 105 & 109 & 0.00 & 6.03 \\
\hline \multirow{2}{*}{ Upper Eyelid Points on Curve } & $\mathrm{Q} 1$ & 91 & 120 & 92 & 120 & -1.10 & 0.00 \\
\cline { 2 - 8 } & $\mathrm{Q} 2$ & 105 & 121 & 102 & 118 & 2.86 & 2.48 \\
\hline \multirow{2}{*}{ Lower Eyelid Control Points } & $\mathrm{P} 1$ & 92 & 136 & 90 & 136 & 2.17 & 0.00 \\
\cline { 2 - 8 } & $\mathrm{P} 2$ & 102 & 134 & 100 & 138 & 1.96 & -2.99 \\
\hline \multirow{2}{*}{ Lower Eyelid Points on Curve } & $\mathrm{Q} 1$ & 92 & 133 & 92 & 134 & 0.00 & -0.75 \\
\cline { 2 - 8 } & $\mathrm{Q} 2$ & 104 & 133 & 102 & 135 & 1.92 & -1.50 \\
\hline
\end{tabular}


Table 4 Coordinate contrast on Right Eye

\begin{tabular}{|c|c|c|c|c|c|c|c|}
\hline \multirow{2}{*}{ Right Eye } & \multicolumn{2}{|c|}{ Manually } & \multicolumn{2}{c|}{ Automatic } & \multicolumn{2}{c|}{ Error[\%] } \\
\cline { 3 - 8 } & & $\mathrm{X}$ & $\mathrm{Y}$ & $\mathrm{X}$ & $\mathrm{Y}$ & $\mathrm{X}$ & $\mathrm{Y}$ \\
\hline \multirow{2}{*}{ Points of the Corner of Eye } & $\mathrm{P}$ & 147 & 132 & 147 & 132 & 0.00 & 0.00 \\
\cline { 2 - 8 } & $\mathrm{P} 3$ & 174 & 130 & 176 & 131 & -1.15 & -0.77 \\
\hline \multirow{2}{*}{ Upper Eyelid Control Points } & $\mathrm{P} 1$ & 155 & 116 & 156 & 117 & -0.65 & -0.86 \\
\cline { 2 - 8 } & $\mathrm{P} 2$ & 164 & 116 & 166 & 114 & -1.22 & 1.72 \\
\hline \multirow{2}{*}{ Upper Eyelid Points on Curve } & $\mathrm{Q} 1$ & 153 & 123 & 157 & 121 & -2.61 & 1.63 \\
\cline { 2 - 8 } & $\mathrm{Q} 2$ & 165 & 121 & 167 & 121 & -1.21 & 0.00 \\
\hline \multirow{2}{*}{ Lower Eyelid Control Points } & $\mathrm{P} 1$ & 157 & 139 & 157 & 137 & 0.00 & 1.44 \\
\cline { 2 - 8 } & $\mathrm{P} 2$ & 165 & 136 & 167 & 139 & -1.21 & -2.21 \\
\hline \multirow{2}{*}{ Lower Eyelid Points on Curve } & $\mathrm{Q} 1$ & 156 & 136 & 157 & 136 & -0.64 & 0.00 \\
\cline { 2 - 8 } & $\mathrm{Q} 2$ & 167 & 134 & 167 & 136 & 0.00 & -1.49 \\
\hline
\end{tabular}

According to the experimental results, it is showed that error of points position obtained automaticlly lies in the acceptable range as for the points on curve or control point obtained by calculation and from the sketch map, so that curve outline fitted automaticlly has a good effect. Through the overall test, all images in the database can fit eye outline automatically and correctly and its error range can be controlled.

\section{Conclusion}

This paper puts forward the method towards automatic extraction of eye outline fitting and curve control point. Based on the feature extraction of Bezier curve and technical route base of facial expression recognition, this method takes Japanese female facial expression database aiming at this testing database, namely JAFFE database, as the test case database. After testing, all images can conduct good outline fitting, and the generated errors also lie in the acceptable range and have no influence on the subsequent expression recognition. The extraction method mentioned in this paper is the automatic extraction and needs not to select database points manually, which lays the foundation for the subsequent automatic facial expression recognition and serves possibilities for real-time facial expression recognition; meanwhile, automatic extraction of features ensures the consistency of database result and reduces errors caused by different operators' operation or unstable operation of the same operator at the time of selecting points.

\section{References}

[1] Ma Tao. Feature Extraction and Facial Expression Recognition Based on Bezier Curve [D]. University of Science and Technology Beijing , 2014.In Chinese.

[2] Tian Hang. Bezier Method Expression of Phase Diagram [D]. University of Science and Technology Beijing, 2013. In Chinese.

[3] Lienhart R, Kuranov A, Pisarevsky V. Empirical Analysis of Detection Cascades of Boosted Classifiers for Rapid Object Detection[M]//Pattern Recognition. Springer Berlin Heidelberg, 2003: 297-304.

[4] Yanjun Li. Research on Face and Features Location Algorithm [D]. North University of China, 2013. In Chinese.

[5] Castrillón M, Déniz O, Guerra C, et al. ENCARA2: Real-time Detection of Multiple Faces at Different Resolutions in Video Streams[J]. Journal of Visual Communication and Image Representation, 2007, 18(2): 130-140.

[6] Ni Kui. Facial Organs Matching and Fusion and Its Application in Facial Animation [D].University of Science and Technology of China, 2009. In Chinese.

[7] Zheng Zhang, Yanping Wang, Guixiang Xue. Digital Image Processing and Machine Vision [J]. 2010. In Chinese

[8] Ren Bin, Wang Bingquan. Threshold and Peak Detection Method Based on Histogram Exponent Smoothing [J]. China Journal of image and Graphics, Volume A, 1997, 2(4): 230-233. 